\title{
The Combined Effect of Electrical Stimulation and High-Load Isometric Contraction on Protein Degradation Pathways in Muscle Atrophy Induced by Hindlimb Unloading
}

\author{
Naoto Fujita, ${ }^{1}$ Shinichiro Murakami, ${ }^{1,2}$ and Hidemi Fujino ${ }^{1}$ \\ ${ }^{1}$ Department of Rehabilitation Science, Graduate School of Health Sciences Kobe University, 7-10-2 Tomogaoka, Suma-Ku, \\ Kobe 654-0142, Japan \\ ${ }^{2}$ Department of Physical Therapy, Himeji Dokkyo University, 7-2-1 Kamiono, Himeji 670-8524, Japan
}

Correspondence should be addressed to Hidemi Fujino, fujino@phoenix.kobe-u.ac.jp

Received 20 May 2011; Revised 1 August 2011; Accepted 1 August 2011

Academic Editor: J.-P. Jin

Copyright (C) 2011 Naoto Fujita et al. This is an open access article distributed under the Creative Commons Attribution License, which permits unrestricted use, distribution, and reproduction in any medium, provided the original work is properly cited.

\begin{abstract}
High-load isometric exercise is considered an effective countermeasure against muscle atrophy, but therapeutic electrical stimulation for muscle atrophy is often performed without loading. In the present study, we investigated the combined effectiveness of electrical stimulation and high-load isometric contraction in preventing muscle atrophy induced by hindlimb unloading. Electrical stimulation without loading resulted in slight attenuation of muscle atrophy. Moreover, combining electrical stimulation with high-load isometric contraction enhanced this effect. In electrical stimulation without loading, inhibition of the overexpression of calpain 1, calpain 2, and MuRF-1 mRNA was confirmed. On the other hand, in electrical stimulation with highload isometric contraction, inhibition of the overexpression of cathepsin L and atrogin-1 mRNA in addition to calpain 1, calpain 2, and MuRF-1 mRNA was confirmed. These findings suggest that the combination of electrical stimulation and high-load isometric contraction is effective as a countermeasure against muscle atrophy.
\end{abstract}

\section{Introduction}

Skeletal muscle atrophy results from a variety of conditions such as hindlimb unloading $[1,2]$, joint immobilization [3, 4], denervation [5, 6], and spinal cord injury [7-9]. Generally resistance exercise training is performed as an effective therapeutic intervention to prevent muscle atrophy induced by these diverse conditions. The effect of resistance exercise is known to be dependent on the intensity of muscle loading [10]. Isometric contraction exercise confers greater protection against muscle atrophy because isometric contraction is a higher-intensity activity than isotonic contraction $[11,12]$. Additionally, muscle contraction using electrical stimulation has also been performed in cases in which it was impossible to perform voluntary limb movement such as denervation and spinal cord injury. Similarly to resistance exercise, the effectiveness of electrically stimulated muscle contraction is influenced by the intensity, including frequency [13, 14], number of contractions [5, 15], and chronaxie [6]. In performing different types of muscle contraction, it is reported that electrical stimulation in isometric contraction is more effective in preventing muscle atrophy than that in isotonic contraction $[7,16]$.

We have investigated the effect of electrical stimulation on muscle atrophy and confirmed that electrical stimulation in isometric contraction can attenuate the decreases in muscle mass and muscle fiber cross-sectional area compared with electrical stimulation in isotonic contraction [17]. Although electrical stimulation in isometric contraction was effective in preventing muscle atrophy, it is still unclear what mechanisms underlie the beneficial effects of electrical stimulation. Three major protein degradation pathways are known to be implicated in muscle atrophy [18]: (1) the lysosomal protease pathway involving cathepsins, which were found to have increased activity in atrophied muscle [19]; (2) the calpain pathway involving calpain 1 and calpain 2 , which are cytosolic calcium-dependent protease that are known to be increased in atrophied muscle [19]; (3) the ubiquitin-proteasome pathway, which involves 2 steps: conjugation of multiubiquitin moieties to the substrate and 
degradation of the tagged protein by the $26 \mathrm{~S}$ proteasome [20]. Muscle-specific ubiquitin ligases, atrogin-1/MAFbx (atrogin-1) and muscle RING finger 1 (MuRF-1), were found to be overexpressed in atrophied muscle [18]. If the relative contributions of the above-mentioned 3 major pathways to enhanced effectiveness of electrical stimulation on muscle atrophy by combined high-load isometric contraction can be verified, this will help establish muscle-specific therapeutic system. The purpose of the present study was to investigate the combined effect of electrical stimulation and high-load isometric contraction to prevent muscle atrophy with a focus on the 3 major protein degradation pathways.

\section{Materials and Methods}

2.1. Experimental Groups. Twenty-one adult male Wistar rats (Japan SLC, Shizuoka, Japan), weighing 282-301 g, were used in the present study. The animals were randomly divided into 4 groups: (1) control (Cont, $n=7$ ), (2) hindlimb unloading ( $\mathrm{HU}, n=7$ ), (3) hindlimb unloading plus electrical stimulation (ES, $n=7$ ), and (4) hindlimb unloading plus the combination of electrical stimulation and highload isometric contraction (ES + IM, $n=7$ ) groups. This study was approved by the Institutional Animal Care and Use Committee and carried out according to the Kobe University Animal Experimentation Regulation. All experiments were conducted in accordance with the National Institute of Health (NIH) Guidelines for the Care and Use of Laboratory Animals (National Research Council, 1996).

2.2. Hindlimb Unloading. Hindlimb unloading was induced in animals by suspending their tails for 14 days, according to the method described by Morey et al. [21]. Briefly, each animal in the HU, ES, and ES + IM groups was fitted with a tail harness and was suspended by a string just high enough to prevent the hindlimbs from bearing weight on the floor or sides of the cage. The forelimbs were allowed to maintain contact with the floor of the cage, and the animals had full access to food and water. The animals in each group were housed in an isolated and environmental controlled room at $22 \pm 2^{\circ} \mathrm{C}$ in a $12 \mathrm{~h}: 12 \mathrm{~h}$ light-dark cycle.

2.3. Electrical Stimulation Protocol. Electrical stimulation began the day after hindlimb unloading and continued for 13 consecutive days. The animals in the ES and ES + IM groups were anesthetized by an intraperitoneal injection of pentobarbital sodium, $40 \mathrm{mg} / \mathrm{kg}$ body weight, during electrical stimulation. The animals in the Cont and HU groups were anesthetized at the same frequency as the ES and ES + IM groups to exclude the influence of the anesthetic. Electrical stimulation equipment (SEN-3301; Nihon Kohden, Tokyo, Japan) that permitted changes in the electrical parameters was used to treat the tibialis anterior muscle percutaneously. Two surface electrodes were used to stimulate the tibialis anterior muscle. One electrode $(5 \mathrm{~cm}$ in diameter) was positioned on the animal's back, and the other active electrode ( $3 \mathrm{~mm}$ in diameter) was adhered on the motor point of the tibialis anterior muscle. During the electrical stimulation, the active electrode was maintained in contact with the skin overlying the tibialis anterior muscle perpendicular to the muscle fibers. The stimulation was a positive square wave with a pulse width of $0.1 \mathrm{~ms}$, and the stimulation pulse amplitude was maintained at $4 \mathrm{~mA}$. Each pulse was delivered at a frequency of $100 \mathrm{~Hz}$. During the electrical stimulation, $1 \mathrm{~s}$ pulses were delivered every $3 \mathrm{~s}$ (time on: $1 \mathrm{~s}$; time off: $2 \mathrm{~s}$ ) for $1 \mathrm{~min}$, followed by $5 \mathrm{~min}$ of rest. Six consecutive stimulation sessions were performed twice in a day, separated by a $9 \mathrm{~h}$ interval. This resulted in a total stimulation duration of $240 \mathrm{~s}$ in a day. In animals that underwent electrical stimulation, that is, ES group, the right tibialis anterior muscle was stimulated without ankle joint fixation to cause an isotonic contraction. Conversely, in the ES + IM group, the left ankle joint was fixed at $90^{\circ}$ in a removable plaster cast during the electrical stimulation to cause high-load isometric contraction. In the rest time between sessions, the plaster cast was removed from the limb.

2.4. Sample Preparation and Histological Analysis. Twelve hours after the last stimulation, all animals were deeply anesthetized by an intraperitoneal injection of sodium pentobarbital, $50 \mathrm{mg} / \mathrm{kg}$ body weight, and then the tibialis anterior muscle was removed and weighed. Thereafter, the animals were sacrificed by an overdose of sodium pentobarbital. Isolated parts from the muscle sample $(\sim 15 \mathrm{mg})$ were kept in RNAlater solution (Ambion, Austin, Tex, USA) for total RNA isolation. The remaining muscle samples were immediately frozen in acetone, cooled in dry ice, and stored at $-80^{\circ} \mathrm{C}$ until histological and western blot analysis.

Serial transverse sections of $10 \mu \mathrm{m}$ thickness were cut on a cryostat (CM-1510S; Leica Microsystems, Mannheim, Germany) from the middle part of the muscle belly in the tibialis anterior muscle at $-25^{\circ} \mathrm{C}$ and mounted on glass slides. The sections were then stained for myofibrillar adenosine triphosphatase (ATPase) at $\mathrm{pH} 4.4$ preincubation to categorize the muscle fiber as type I, IIA, or IIB on the basis of a previous study [22]. The sections stained by ATPase were used to determine the composition of muscle fiber types and to measure cross-sectional areas of each muscle fiber type. The sections were also stained for succinate dehydrogenase (SDH). Each muscle fiber was matched for ATPase and SDH stains, and fibers found to be lightly stained with ATPase and those darkly stained with SDH were omitted from the measurement of muscle fiber crosssectional area. A measuring field was set over the entire muscle cross-section for the determination of muscle fiber type composition. At least 100 randomly selected crosssectional areas of each muscle fiber type were investigated. The sections were measured using the ImageJ software program (NIH, Maryland, USA).

2.5. Western Blot Analysis. The frozen muscle samples were homogenized in ice-cold homogenizing buffer containing $20 \mathrm{mM}$ Tris- $\mathrm{HCl}, \mathrm{pH}$ 7.4, $25 \mathrm{mM} \mathrm{KCl}, 5 \mathrm{mM}$ EDTA, $5 \mathrm{mM}$ EGTA, $1 \mathrm{mM}$ Dithiothreitol, and protease inhibitor cocktail (Nacalai tesque, Kyoto, Japan). The homogenates were centrifuged at $15,000 \mathrm{~g}$ for $25 \mathrm{~min}$ at $4^{\circ} \mathrm{C}$. Total protein 
TABLE 1: Body weight $(\mathrm{g})$, wet weight ( $\mathrm{mg})$ of the tibialis anterior muscle, and ratio of wet weight to body weight (mg/g).

\begin{tabular}{|c|c|c|c|c|}
\hline & Cont & $\mathrm{HU}$ & ES & $\mathrm{ES}+\mathrm{IM}$ \\
\hline Body weight, g & $308 \pm 3$ & $246 \pm 5^{*}$ & $238 \pm 3^{*}$ & $238 \pm 3^{*}$ \\
\hline Muscle wet weight, mg & $539 \pm 6$ & $418 \pm 4^{*}$ & $429 \pm 12^{*}$ & $478 \pm 12^{* \dagger \ddagger}$ \\
\hline Muscle wet weight to body ratio, $\mathrm{mg} / \mathrm{g}$ & $1.74 \pm 0.02$ & $1.70 \pm 0.02$ & $1.79 \pm 0.03$ & $2.00 \pm 0.02^{* \dagger \dagger}$ \\
\hline
\end{tabular}

Values are the mean \pm SEM. Cont: control group $(n=14)$; HU: hindlimb unloading for 14 days group $(n=14)$; ES: hindlimb unloading plus electrical stimulation group ( $n=7)$; ES + IM: hindlimb unloading plus the combination of electrical stimulation and isometric contraction group $(n=7) .{ }^{\dagger}$, and ${ }^{\ddagger}$ are significantly different from the Cont, $\mathrm{HU}$, and ES groups, respectively, at $P<0.05$.

concentration was determined by the use of a protein determination kit (BioRad, Calif, USA). The homogenates were solubilized in sample loading buffer containing $50 \mathrm{mM}$ Tris- $\mathrm{HCl}, \mathrm{pH} 6.8,2 \%$ sodium dodecyl sulfate, $10 \%$ glycerol, $5 \% \beta$-mercaptoethanol, and $0.005 \%$ bromophenol blue. The samples were boiled for $5 \mathrm{~min}$ at $60^{\circ} \mathrm{C}$. Twenty micrograms of sample protein was subjected to SDS-PAGE and then transferred to PVDF membrane. Following overnight blocking step in $0.3 \%$ skimmed milk in PBST, the membranes were incubated with anticathepsin L (Abcam, Tokyo, Japan), anticalpain 1 (Cell Signaling, Danvers, Mass, USA), anticalpain 2 (Cell Signaling, Danvers, Mass, USA), and antiubiquitin (Stressgen, Plymouth Meeting, Pa, USA) at $4^{\circ} \mathrm{C}$. Following overnight incubation, the membranes were incubated for $60 \mathrm{~min}$ at room temperature with anti-rabbit or anti-mouse IgG conjugated to horseradish peroxidase (GE Healthcare, Amersham, NJ, USA). The signals were detected using a chemiluminescent (ECL plus, GE Healthcare, Amersham, NJ, USA) and analyzed with an image reader (LAS-1000, Fujifilm, Tokyo, Japan).

2.6. Real-Time Quantitative Polymerase Chain Reaction (qPCR) Analysis. Total RNA was extracted from $\sim 10 \mathrm{mg}$ of each muscle using an extraction kit (QuickGene RNA tissue kit SII; Fujifilm, Tokyo, Japan). Reverse transcription was carried out using the High Capacity cDNA Archive Kit (Applied Biosystems, Foster City, Calif, USA), and then cDNA samples were stored at $-20^{\circ} \mathrm{C}$.

Expression levels of cathepsin L (Rn00565793_m1), calpain 1 (Rn00569689_m1), calpain 2 (Rn00567422_m1), atrogin-1 (Rn00591730_m1), and MuRF-1 (Rn00590197_m1) mRNA were quantified by TaqMan Gene Expression Assays (Applied Biosystems). Each TaqMan probe and primer set was validated by performing QPCR with a series of cDNA template dilutions to obtain standard curves of threshold cycle time against relative concentration using the normalization gene 18S. qPCR was performed using PCR Fast Universal Master Mix (Applied Biosystems) in a MicroAmp 96-well reaction plate. Each well contained $1 \mu \mathrm{L}$ cDNA template, $10 \mu \mathrm{L}$ PCR Fast Master Mix, $8 \mu \mathrm{L}$ RNasefree water, and $1 \mu \mathrm{L}$ TaqMan Gene Expression Assays in a reaction volume of $20 \mu \mathrm{L}$. All samples and nontemplate control reactions were performed in a 7500 Fast Sequence Detection System (Applied Biosystems) at $50^{\circ} \mathrm{C}$ for $2 \mathrm{~min}$, $95^{\circ} \mathrm{C}$ for $10 \mathrm{~min}$, followed by 40 cycles at $95^{\circ} \mathrm{C}$ for $15 \mathrm{~s}$ and $60^{\circ} \mathrm{C}$ for $1 \mathrm{~min}$.

2.7. Statistical Analysis. The data are expressed as mean \pm SEM. Significant differences between the 4 experimental groups were analyzed using one-way analysis of variance followed by Tukey HSD post hoc test. Statistical significance was set at $P<0.05$.

\section{Results}

3.1. Muscle Wet Weight. Body weight, muscle wet weight, and ratio of muscle wet weight to body weight are shown in Table 1. The values of body weight in the HU, ES, and $\mathrm{HU}+\mathrm{IM}$ groups were significantly lower than that in the Cont group. The values of muscle wet weight in the HU, ES, and ES + IM groups were significantly lower than that in the Cont group. The value of muscle wet weight in the ES + IM group was significantly larger than that in the HU group, whereas there was no significant difference between the ES and HU groups. The value of muscle wet weight in the ES + IM group was significantly larger than that in the ES group. For the ratio of muscle wet weight to body weight, the value in the ES + IM group was significantly larger than that in the HU and ES groups.

3.2. Muscle Fiber Cross-Sectional Area. ATPase staining revealed that the tibialis anterior muscles were composed of type I (0.3-2.2\%), type IIA (3.4-5.8\%), and type IIB (92.4-96.2\%) fibers (Figure 1), and there were no significant differences between the 4 groups. The sections in the ES and ES + IM groups did not have any histological signs of skeletal muscle injury.

The mean values of the cross-sectional area of the type I fibers in the HU and ES groups were significantly less than that in the Cont group, whereas there were no significant differences between the Cont and ES + IM groups (Figure 2(a)). The values in the ES and ES + IM groups were significantly larger than that in the HU group. The value in the ES + IM group was significantly larger than that in the ES group. In both the type IIA (Figure 2(b)) and IIB (Figure 2(c)) fibers, the mean fiber cross-sectional area values in the HU, ES, and ES + IM groups were significantly less than that in the Cont group. The mean values in the ES and ES + IM groups were significantly larger than that in the $\mathrm{HU}$ group, and the mean value in the ES + IM group was significantly larger than in the ES group.

3.3. Lysosomal Protease Pathway. For the expression level of cathepsin L protein, there were no significant differences between the 4 groups (Figure 3(a)). However, the expression level of cathepsin L mRNA in the HU group was increased by $2.25 \pm 0.19$-fold compared with the Cont group 


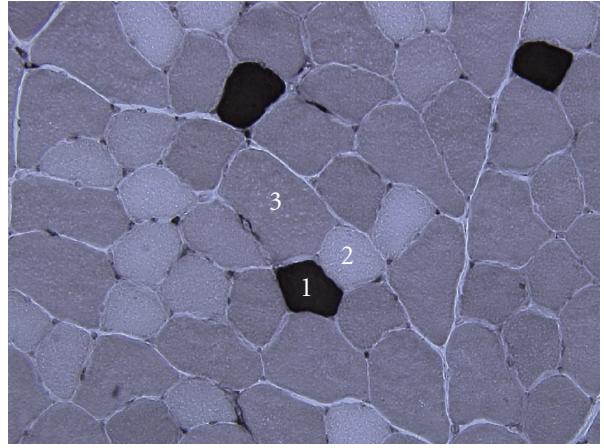

(a)

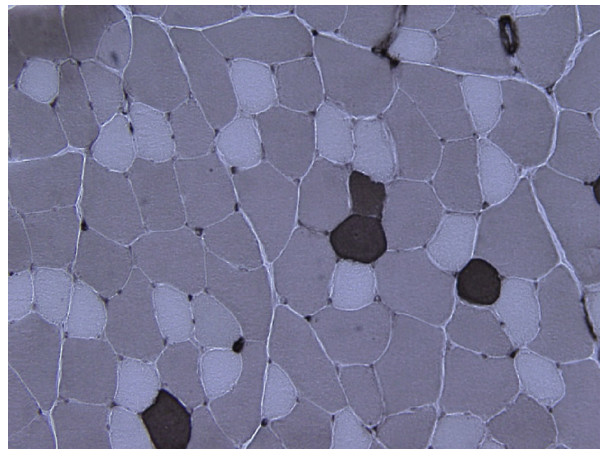

(c)

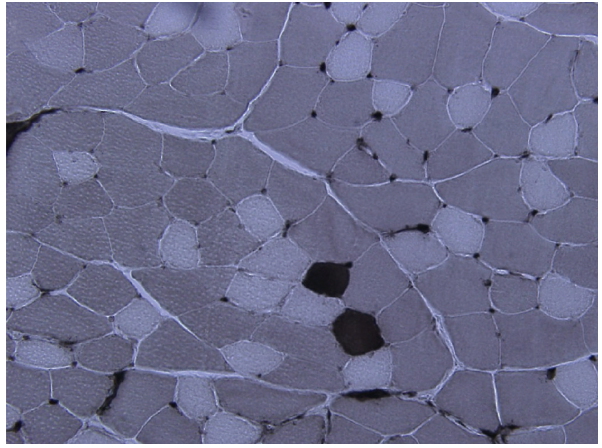

(b)

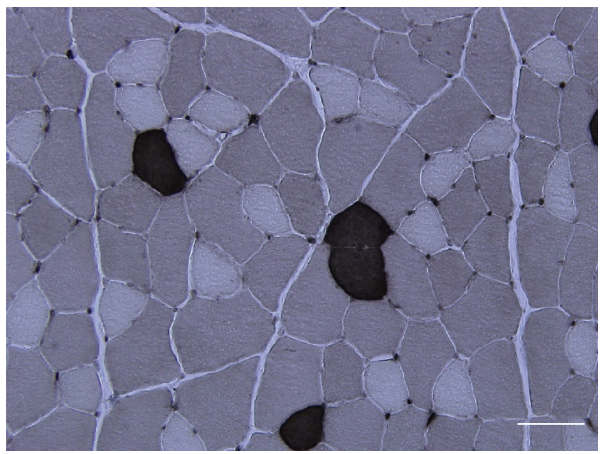

(d)

FIgURE 1: Transverse sections of the tibialis anterior muscle pretreated at $\mathrm{pH} 4.4$ were assayed for myofibrillar ATPase staining. (a) Cont group; (b) HU group; (c) ES group; (d)ES + IM group. 1: type I; 2: type IIA; 3: type IIB. Bar = $50 \mu \mathrm{m}$.

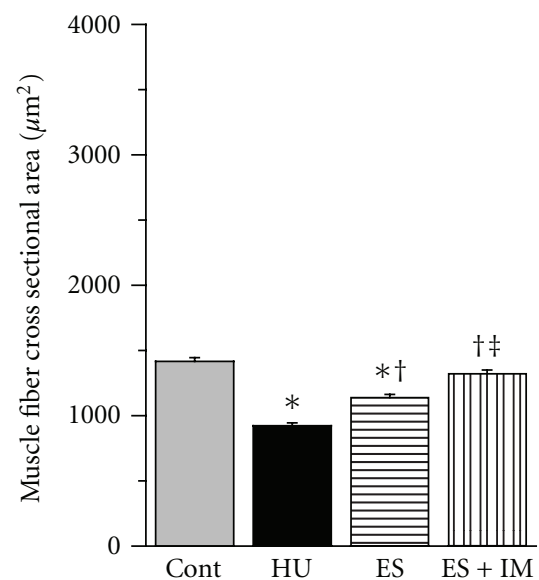

(a)

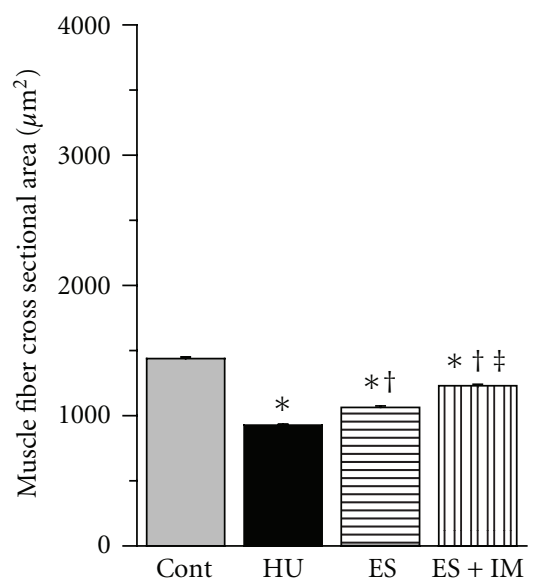

(b)

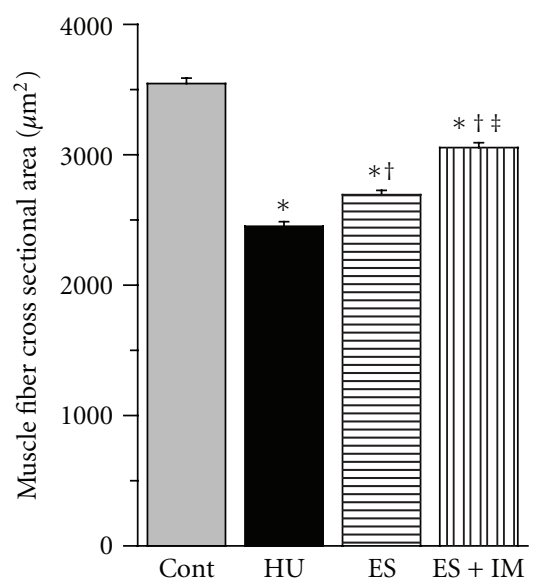

(c)

FIgURE 2: Cross-sectional area $\left(\mu \mathrm{m}^{2}\right)$ of type I (a), type IIA (b), and type IIB (c) fibers in the tibialis anterior muscle. Values are presented as the mean \pm SEM. ${ }^{*},{ }^{\dagger}$, and ${ }^{\ddagger}$ are significantly different from the Cont, HU, and ES groups, respectively, at $P<0.05$. In each muscle fiber type, over 100 muscle fibers were measured.

(Figure 4(a)). There were no significant differences between the ES and HU groups. In contrast, the value in the ES + IM group was significantly less than that in the HU and ES groups and was maintained at the control group level.

3.4. Calpain Pathway. For the expression level of calpain 1 (Figure 3(b)) and calpain 2 (Figure 3(c)) proteins, the values in the HU group were significantly higher than that in the Cont group. The values in the ES and ES + IM groups were significantly less than that in the HU group. However, there were no significant differences between the ES and ES + IM groups.

The expression levels of calpain 1 (Figure 4(b)) and calpain 2 (Figure 4(c)) mRNA in the HU group increased by 


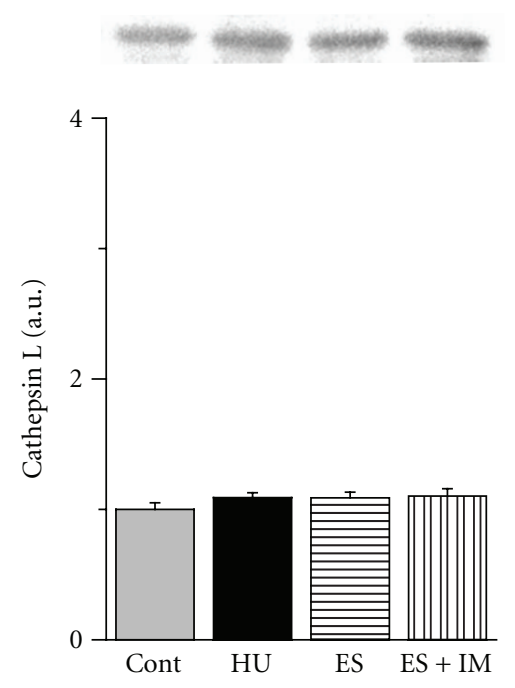

(a)

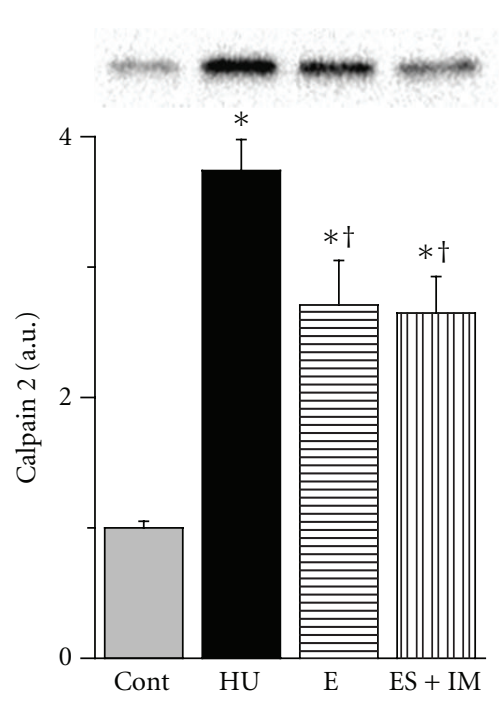

(c)

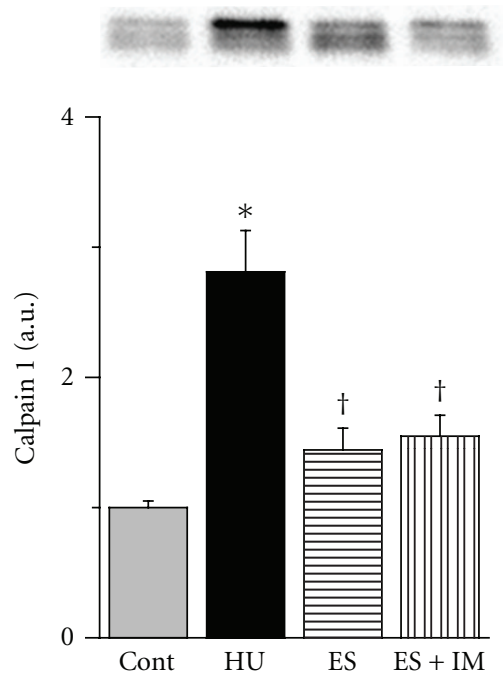

(b)

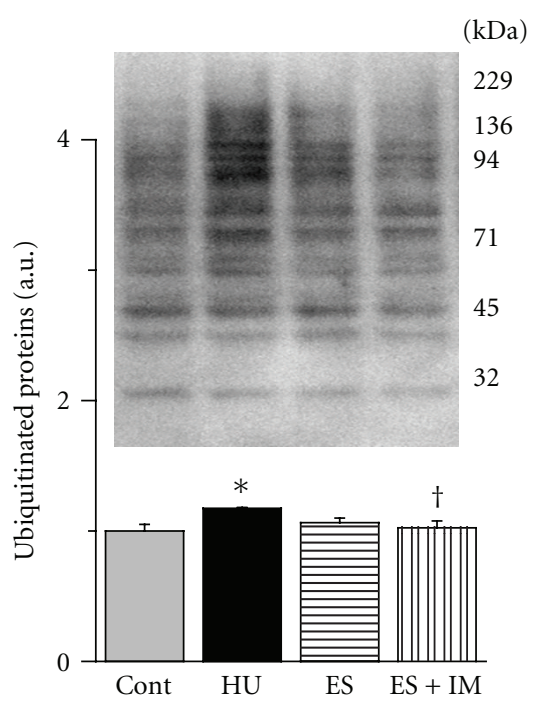

(d)

FIGURE 3: Expression levels of cathepsin L (a), calpain 1 (b), calpain 2 (c), and ubiquitinated proteins (d) in the tibialis anterior muscle and representative western blot. Values were calculated as the fold changes relative to the Cont and presented as the mean \pm SEM. ${ }^{*}$ and ${ }^{\dagger}$ are significantly different from the Cont and HU groups, respectively, at $P<0.05 . N=14$ in the Cont and HU groups, $n=7$ in the ES and ES + IM groups.

$1.28 \pm 0.09$-fold and $2.5 \pm 0.21$-fold compared with the Cont group, respectively. The levels of both calpain 1 and calpain 2 mRNA in the ES and ES + IM groups were significantly less than that in the HU. There were no significant differences between the ES and ES + IM groups.

3.5. Ubiquitin-Proteasome Pathway. For the expression level of ubiquitinated proteins, the value in the HU group was significantly higher than that in the Cont group (Figure 3(d)). Although there were no significant differences between the ES and HU groups, the value in the ES + IM group was significantly less than that in the HU group.
The expression level of atrogin-1 mRNA in the HU group increased by $2.71 \pm 0.37$-fold compared with the Cont group (Figure $4(\mathrm{~d})$ ). Although there were no significant differences between the ES and HU groups, the value in the ES + IM group was significantly less than that in the HU group. The value in the ES + IM group appeared to be less than that in the ES group $(P=0.0514)$ and was maintained at the control group level. The expression level of MuRF-1 (Figure 4(e)) mRNA in the HU group increased by $1.83 \pm$ 0.29 -fold compared with the Cont group. The values in the ES and ES + IM groups were significantly less than that in the HU group. There were no significant differences among the Cont, ES, and ES + IM groups. 


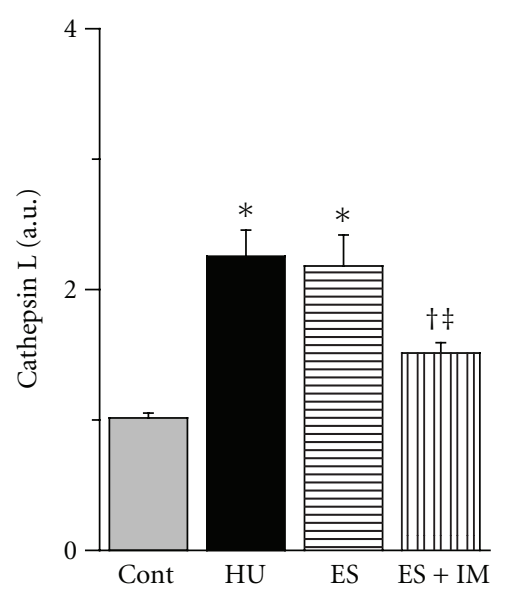

(a)

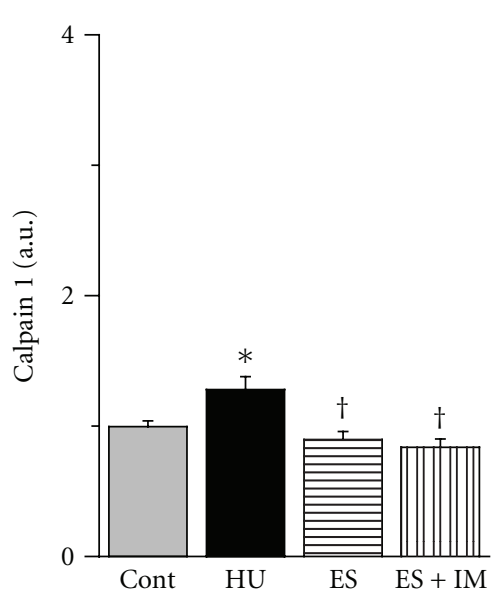

(b)

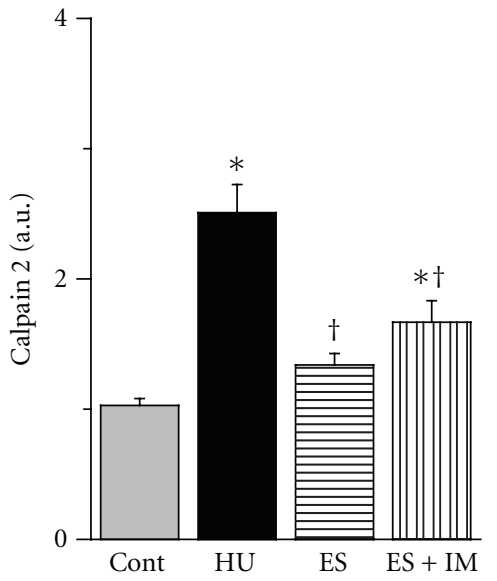

(c)

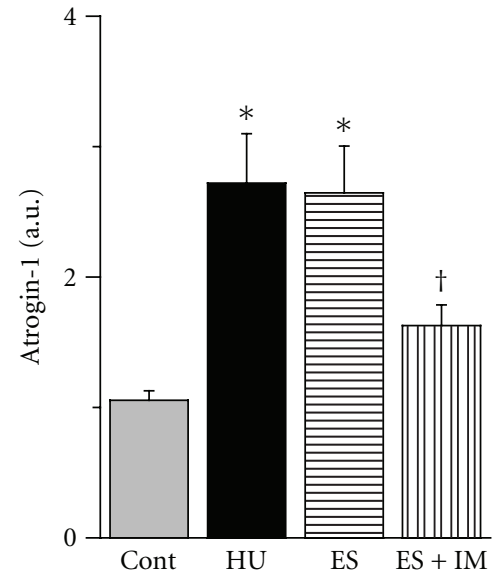

(d)

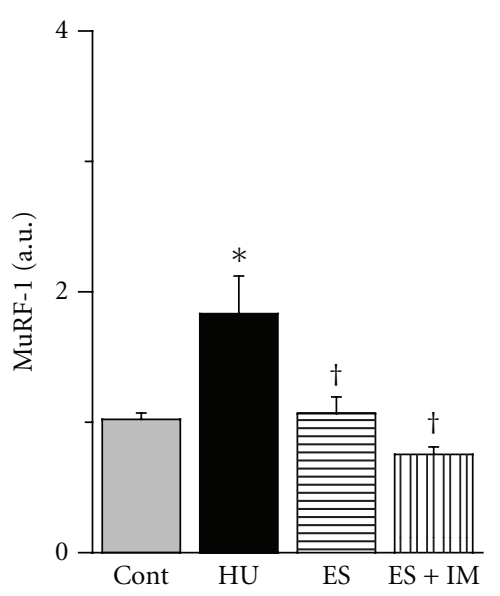

(e)

FIGURE 4: Expression levels of cathepsin L (a), calpain 1 (b), calpain 2 (c), atrogin-1/MAFbx (atrogin-1; d), and muscle RING finger-1 (MuRF-1; e) mRNA in the tibialis anterior muscle. Values were calculated as the fold changes relative to the Cont and presented as the mean \pm SEM. ${ }^{*},{ }^{\dagger}$, and ${ }^{\ddagger}$ are significantly different from the Cont, HU, and ES groups, respectively, at $P<0.05 . N=14$ in the Cont and HU groups, $n=7$ in the ES and ES + IM groups.

\section{Discussion}

In the present study, hindlimb unloading resulted in muscle atrophy of the tibialis anterior. Skeletal muscle atrophy is characterized by decreased protein synthesis and increased protein degradation. The decrease in protein synthesis reaches a peak within a few days after the start of unloading, whereas the increase in protein degradation reaches a peak 14 days after unloading [23]. Because hindlimb unloading was applied for 14 days in the present study, it was hypothesized that the increase in protein degradation was related closely rather than the decrease in protein synthesis to the atrophied tibialis anterior muscle. Three major protein degradation pathways are implicated in skeletal muscle atrophy resulting from a variety of disuse conditions (e.g., hindlimb unloading, immobilization, denervation, and spinal cord injury): the lysosomal protease pathway, the cytosolic calciumdependent calpain pathway, and the ubiquitin-proteasome pathway [18]. In the present study, the expression level of cathepsin L mRNA, one of the lysosomal proteases induced by hindlimb unloading [19], was increased by 2.25 -fold in the HU group. Furthermore, hindlimb unloading evoked the overexpression of calpain $1(1.28$-fold) and calpain 2 (2.5-fold) mRNA. As for skeletal muscle atrophy-related ubiquitin ligases, the levels of atrogin-1 and MuRF-1 mRNA were increased by 2.71 -fold and 1.83-fold, respectively, with hindlimb unloading. Therefore, it can be postulated that the decreases in muscle mass and muscle fiber cross-sectional areas in all types in the HU group were due to the activation of the 3 major protein degradation pathways.

Electrical stimulation in isotonic contraction in the ES group attenuated the decrease in the cross-sectional areas in all muscle fiber types induced by hindlimb unloading. Almost all results in the present study were consistent with those of Boonyarom et al. [14]. In the present study, the electrical stimulation in isotonic contraction was performed percutaneously during hindlimb unloading for 14 days. The inhibition of overexpression of calpain 1, calpain 2, and 
MuRF-1 mRNA in the ES group may have contributed to the attenuation of muscle atrophy observed. However, the preventive effect of electrical stimulation on muscle atrophy in the ES group was not enough compared with the ES + IM group. Taillandier et al. [19] have reported that the inhibition of the calpain pathway failed to suppress protein degradation in atrophied muscle by unloading. In addition, the assembly and scaffolding of myofibrillar proteins such as nebulin, titin, and vinculin is known as calpains substrates [24], and the calpains are unable to degrade actin and myosin [25]. Because the most abundant proteins in muscle fiber are actin and myosin [18], the inhibition of calpains in atrophied muscle may have only a very little effect on the crosssectional area of muscle fiber. Therefore, though this is just our speculation, the effect of electrical stimulation on muscle atrophy in the ES group was likely not based on the diminished expression of calpains mRNA, but that of MuRF-1.

The combination of electrical stimulation and high-load isometric contraction in the ES + IM group was more effective in preventing muscle atrophy than electrical stimulation in isotonic contraction in the ES group. Although the expression of ubiquitinated proteins in the ES group was of the same level as the HU group, the value in the ES + IM group was significantly less than that in the HU group. In the ES + IM group, there was inhibition of overexpression of cathepsin L and atrogin-1 mRNA in addition to calpain 1, calpain 2, and MuRF-1. Collectively, these results were contributive to the enhanced effect of electrical stimulation on muscle atrophy. However, it is reported that cathepsins are unable to degrade myofibrillar proteins; their major substrates are membrane proteins such as receptors, ligands, channels, and transporters [26]. Additionally, some studies have reported that, when lysosomal activity is inhibited, myofibrillar protein degradation rates are only slightly reduced $[27,28]$. Therefore, the major factor underlying the enhanced preventive effect on muscle atrophy in the ES + IM group might not be diminished cathepsin L mRNA expression. Many previous studies have indicated that protein degradation during unloading occurs principally through the ubiquitin-proteasome pathway $[18,29,30]$; with respect to the ubiquitin-proteasome pathway in the ES+ IM group, not only overexpression of MuRF-1 mRNA but also that of atrogin-1 was inhibited. Previous studies with MuRF-1 knockout mice have shown that the decrease in the muscle fiber cross-sectional area induced by hindlimb unloading [31] and denervation [32] were unable to prevent completely. Furthermore, Bodine et al. [33] compared atrogin-1 knockout and MuRF-1 knockout mice to show that the preventive effect on muscle atrophy is prominent in atrogin-1 knockout rather than in MuRF-1 knockout [33]. Therefore, there is a possibility that the differences between the ES and ES+ IM groups are due to whether overexpression of both atrogin-1 and MuRF-1 mRNA was inhibited. However, this is just speculation, so there is a need for further study to show the evidence supporting this speculation.

\section{Conclusion}

Although electrical stimulation in isotonic contraction slightly attenuated muscle atrophy induced by hindlimb unloading, the combination of electrical stimulation and high-load isometric contraction inhibited increases in all 3 protein degradation pathways linked to muscle atrophy and enhanced the effect of electrical stimulation to prevent muscle atrophy. Electrical stimulation in isotonic contraction attenuated only MuRF-1 mRNA overexpression, whereas the combination of electrical stimulation and high-load isometric contraction inhibited both atrogin- 1 and MuRF- 1 mRNA overexpression. The result of the electrical stimulation in isotonic contraction in this study is consistent with that of a previous study [34]. Macpherson et al. [34] investigated the effect of electrical stimulation in isotonic contraction on expression of the ubiquitin ligases in denervated muscle and showed that overexpression of MuRF-1 mRNA is relatively more inhibited than that of atrogin- 1 . Therefore, the findings of the present study suggest that when high-load isometric contraction is added to electrical stimulation, overexpression of not only MuRF-1 mRNA but also atrogin-1 is inhibited during unloading. It is still unknown what the differences are between the roles of atrogin-1 and MuRF-1 in the ubiquitin-proteasome pathway, and this requires further study. However, the combination of electrical stimulation and high-load isometric contraction is promising as an effective therapeutic intervention.

\section{Acknowledgments}

This study was supported by The Japanese Society of Physio Therapeutics and Grants-in-Aid for Scientific Research from the Japanese Ministry of Education, Culture, Sports, Science, and Technology.

\section{References}

[1] H. Fujino, A. Ishihara, S. Murakami et al., "Protective effects of exercise preconditioning on hindlimb unloading-induced atrophy of rat soleus muscle," Acta Physiologica, vol. 197, no. 1, pp. 65-74, 2009.

[2] I. Takeda, H. Fujino, S. Murakami, H. Kondo, F. Nagatomo, and A. Ishihara, "Thermal preconditioning prevents fiber type transformation of the unloading induced-atrophied muscle in rats," Journal of Muscle Research and Cell Motility, vol. 30, no. 3-4, pp. 145-152, 2009.

[3] N. Fujita, T. Fujimoto, H. Tasaki, T. Arakawa, T. Matsubara, and A. Miki, "Influence of muscle length on muscle atrophy in the mouse tibialis anterior and soleus muscles," Biomedical Research, vol. 30, no. 1, pp. 39-45, 2009.

[4] N. Fujita, T. Arakawa, T. Matsubara, H. Ando, and A. Miki, "Influence of fixed muscle length and contractile properties on atrophy and subsequent recovery in the rat soleus and plantaris muscles," Archives of Histology and Cytology, vol. 72, no. 3, pp. 151-163, 2009.

[5] D. E. Dow, P. S. Cederna, C. A. Hassett, T. Y. Kostrominova, J. A. Faulkner, and R. G. Dennis, "Number of contractions to maintain mass and force of a denervated rat muscle," Muscle and Nerve, vol. 30, no. 1, pp. 77-86, 2004.

[6] T. L. Russo, S. M. Peviani, C. M. Freria, D. Gigo-Benato, S. Geuna, and T. F. Salvini, "Electrical stimulation based on chronaxie reduces atrogin-1 and myod gene expressions in 
denervated rat muscle," Muscle and Nerve, vol. 35, no. 1, pp. 87-97, 2007.

[7] R. R. Roy, H. Zhong, J. A. Hodgson et al., "Influences of electromechanical events in defining skeletal muscle properties," Muscle and Nerve, vol. 26, no. 2, pp. 238-251, 2002.

[8] S. J. Kim, R. R. Roy, H. Zhong et al., "Electromechanical stimulation ameliorates inactivity-induced adaptations in the medial gastrocnemius of adult rats," Journal of Applied Physiology, vol. 103, no. 1, pp. 195-205, 2007.

[9] S. J. Kim, R. R. Roy, J. A. Kim et al., "Gene expression during inactivity-induced muscle atrophy: effects of brief bouts of a forceful contraction countermeasure," Journal of Applied Physiology, vol. 105, no. 4, pp. 1246-1254, 2008.

[10] K. J. Milne and E. G. Noble, "Exercise-induced elevation of HSP70 is intensity dependent," Journal of Applied Physiology, vol. 93, no. 2, pp. 561-568, 2002.

[11] R. H. Fitts, "Effects of regular exercise training on skeletal muscle contractile function," American Journal of Physical Medicine and Rehabilitation, vol. 82, no. 4, pp. 320-331, 2003.

[12] J. E. Hurst and R. H. Fitts, "Hindlimb unloading-induced muscle atrophy and loss of function: protective effect of isometric exercise," Journal of Applied Physiology, vol. 95, no. 4, pp. 1405-1417, 2003.

[13] A. Misawa, Y. Shimada, T. Matsunaga, and K. Sato, "The effects of therapeutic electric stimulation on acute muscle atrophy in rats after spinal cord injury," Archives of Physical Medicine and Rehabilitation, vol. 82, no. 11, pp. 1596-1603, 2001.

[14] O. Boonyarom, N. Kozuka, K. Matsuyama, and S. Murakami, "Effect of electrical stimulation to prevent muscle atrophy on morphologic and histologic properties of hindlimb suspended rat hindlimb muscles," American Journal of Physical Medicine and Rehabilitation, vol. 88, no. 9, pp. 719-726, 2009.

[15] R. G. Dennis, D. E. Dow, and J. A. Faulkner, "An implantable device for stimulation of denervated muscles in rats," Medical Engineering and Physics, vol. 25, no. 3, pp. 239-253, 2003.

[16] F. Haddad, G. R. Adams, P. W. Bodell, and K. M. Baldwin, "Isometric resistance exercise fails to counteract skeletal muscle atrophy processes during the initial stages of unloading," Journal of Applied Physiology, vol. 100, no. 2, pp. 433-441, 2006.

[17] N. Fujita, S. Murakami, T. Arakawa, A. Miki, and H. Fujino, "The combined effect of electrical stimulation and resistance isometric contraction on muscle atrophy in rat tibialis anterior muscle," Bosnian Journal of Basic Medical Sciences, vol. 11, no. 2, pp. 74-79, 2011.

[18] R. W. Jackman and S. C. Kandarian, "The molecular basis of skeletal muscle atrophy," American Journal of Physiology-Cell Physiology, vol. 287, no. 4, pp. C834-C843, 2004.

[19] D. Taillandier, E. Aurousseau, D. Meynial-Denis et al., "Coordinate activation of lysosomal, Ca2+-activated and ATPubiquitin-dependent proteinases in the unweighted rat soleus muscle," Biochemical Journal, vol. 316, part 1, pp. 65-72, 1996.

[20] M. H. Glickman and A. Ciechanover, "The ubiquitinproteasome proteolytic pathway: destruction for the sake of construction," Physiological Reviews, vol. 82, no. 2, pp. 373428, 2002.

[21] E. R. Morey, E. E. Sabelman, R. T. Turner, and D. J. Baylink, "A new rat model simulating some aspects of space flight," Physiologist, vol. 22, no. 6, pp. S23-S24, 1979.
[22] K. Punkt, A. Naupert, and G. Asmussen, "Differentiation of rat skeletal muscle fibres during development and ageing," Acta Histochemica, vol. 106, no. 2, pp. 145-154, 2004.

[23] D. B. Thomason and F. W. Booth, "Atrophy of the soleus muscle by hindlimb unweighting," Journal of Applied Physiology, vol. 68, no. 1, pp. 1-12, 1990.

[24] J. Huang and N. E. Forsberg, "Role of calpain in skeletalmuscle protein degradation," Proceedings of the National Academy of Sciences of the United States of America, vol. 95, no. 21, pp. 12100-12105, 1998.

[25] M. G. Thompson and R. M. Palmer, "Signalling pathways regulating protein turnover in skeletal muscle," Cellular Signalling, vol. 10, no. 1, pp. 1-11, 1998.

[26] R. J. Mayer, "The meteoric rise of regulated intracellular proteolysis," Nature Reviews Molecular Cell Biology, vol. 1, no. 2, pp. 145-148, 2000.

[27] K. Furuno and A. L. Goldberg, "The activation of protein degradation in muscle by $\mathrm{Ca} 2+$ or muscle injury does not involve a lysosomal mechanism," Biochemical Journal, vol. 237, no. 3, pp. 859-864, 1986.

[28] M. E. Tischler, S. Rosenberg, S. Satarug et al., "Different mechanisms of increased proteolysis in atrophy induced by denervation or unweighting of rat soleus muscle," Metabolism, vol. 39, no. 7, pp. 756-763, 1990.

[29] S. H. Lecker, V. Solomon, W. E. Mitch, and A. L. Goldberg, "Muscle protein breakdown and the critical role of the ubiquitin-proteasome pathway in normal and disease states," Journal of Nutrition, vol. 129, supplement 1, pp. 227S-237S, 1999.

[30] R. T. Jagoe and A. L. Goldberg, "What do we really know about the ubiquitin-proteasome pathway in muscle atrophy?" Current Opinion in Clinical Nutrition and Metabolic Care, vol. 4, no. 3, pp. 183-190, 2001.

[31] S. Labeit, C. H. Kohl, C. C. Witt, D. Labeit, J. Jung, and H. Granzier, "Modulation of muscle atrophy, fatigue and MLC phosphorylation by MuRF1 as indicated by hindlimb suspension studies on MuRF1-KO mice," Journal of Biomedicine and Biotechnology, vol. 2010, Article ID 693741, 2010.

[32] A. S. Moriscot, I. L. Baptista, J. Bogomolovas et al., "MuRF1 is a muscle fiber-type II associated factor and together with MuRF2 regulates type-II fiber trophicity and maintenance," Journal of Structural Biology, vol. 170, no. 2, pp. 344-353, 2010.

[33] S. C. Bodine, E. Latres, S. Baumhueter et al., "Identification of ubiquitin ligases required for skeletal muscle atrophy," Science, vol. 294, no. 5547, pp. 1704-1708, 2001.

[34] P. C. D. Macpherson, X. Wang, and D. Goldman, "Myogenin regulates denervation-dependent muscle atrophy in mouse soleus muscle," Journal of Cellular Biochemistry, vol. 112, no. 8, pp. 2149-2159, 2011. 

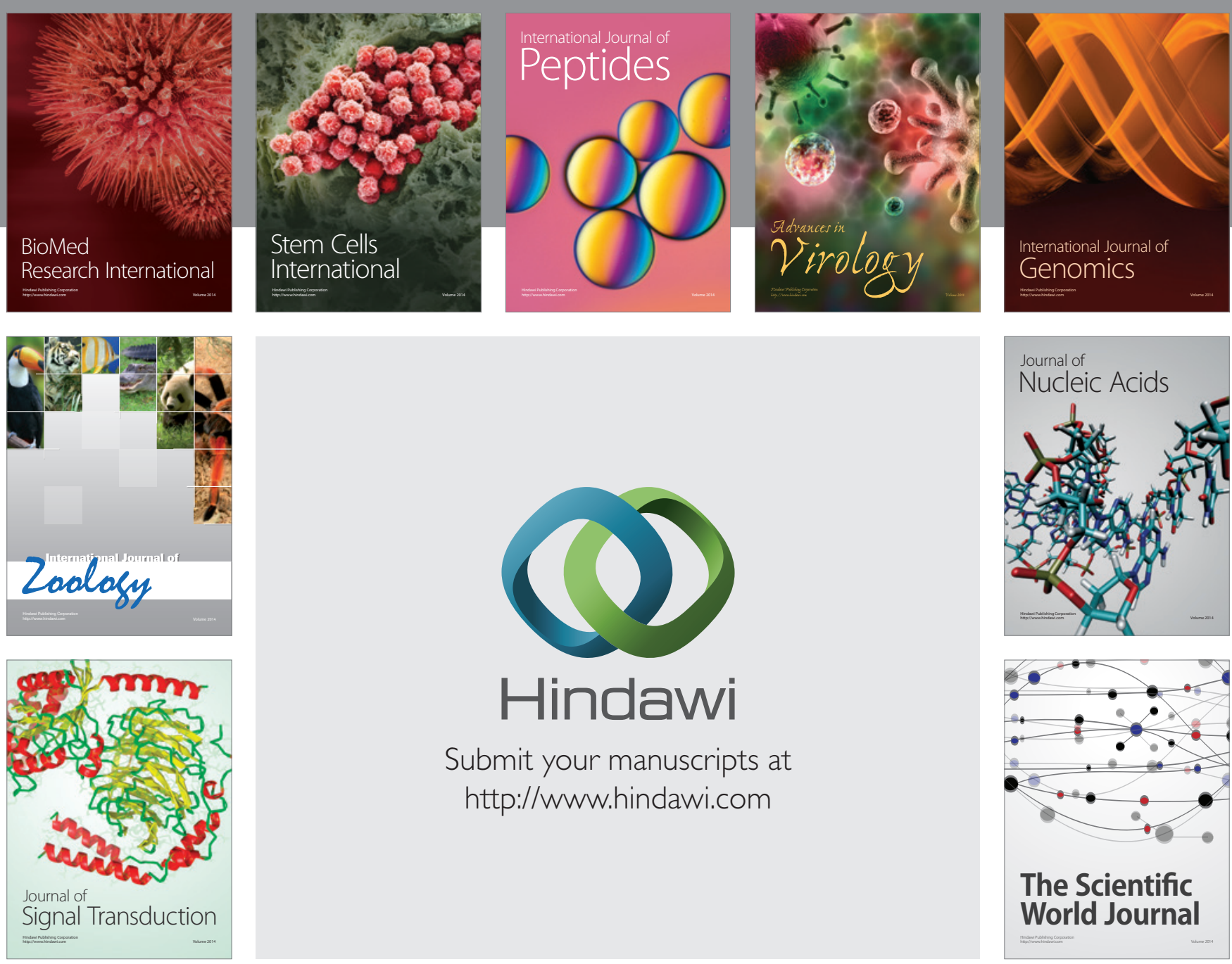

Submit your manuscripts at

http://www.hindawi.com
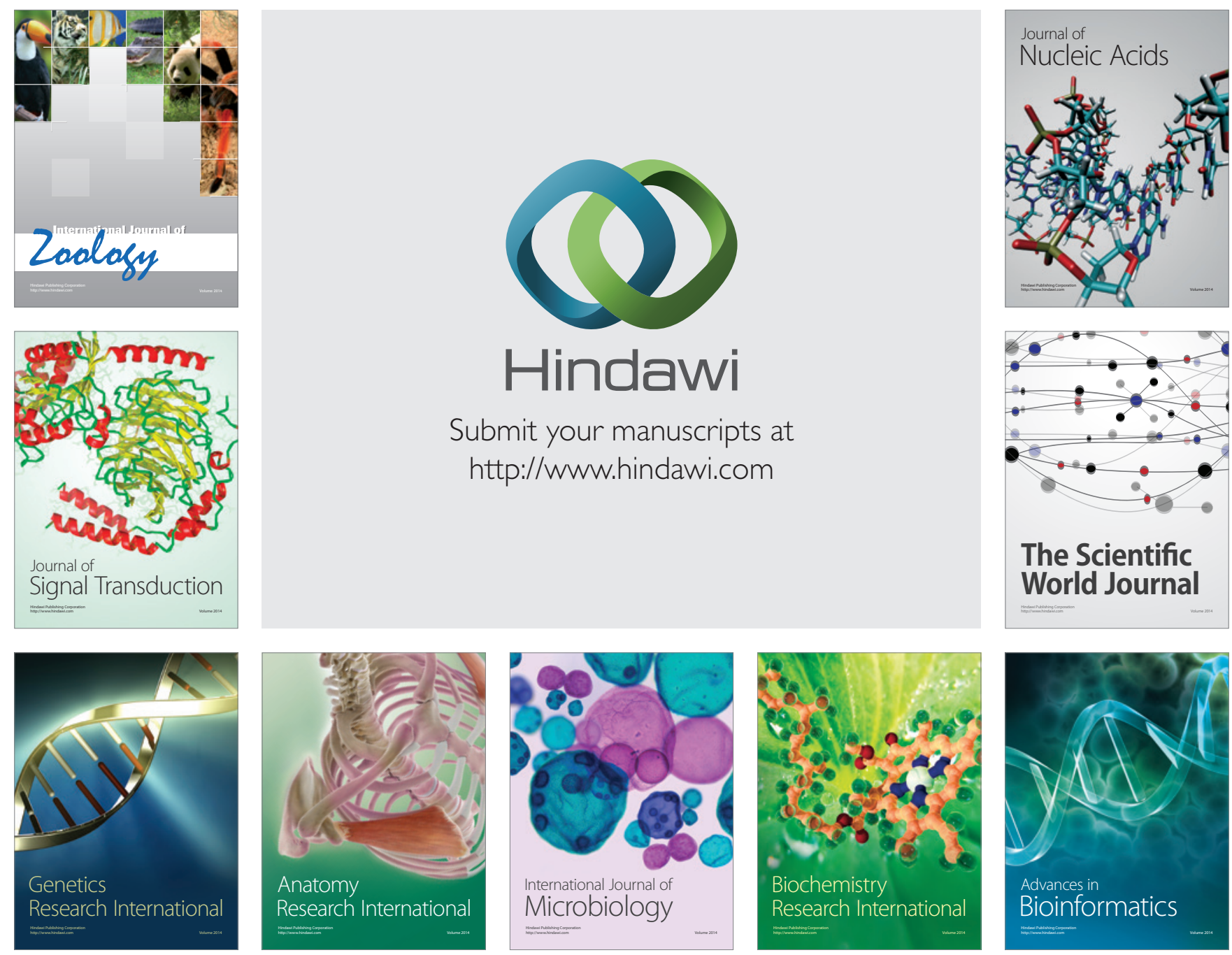

The Scientific World Journal
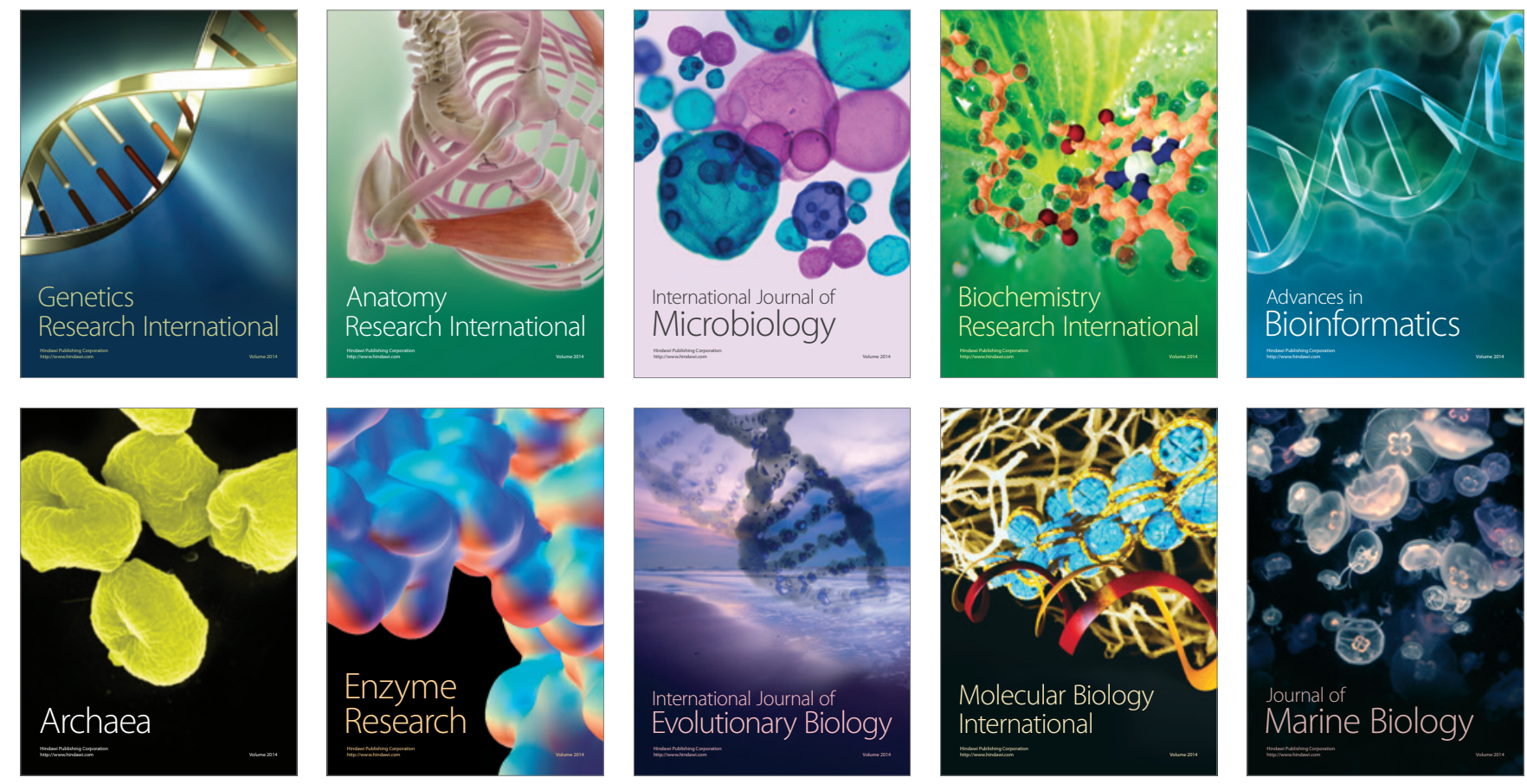